\title{
POLIURETANOS ELASTOMÉRICOS OBTENIDOS A PARTIR DE ACEITE DE RICINO Y ALMIDÓN DE YUCA ORIGINAL Y MODIFICADO CON ANHÍDRIDO PROPIÓNICO: SÍNTESIS, PROPIEDADES FISICOQUÍMICAS Y FISICOMECÁNICAS
}

\author{
Manuel F. Valero* \\ Facultad de Ingeniería, Universidad de la Sabana, Campus Universitario del Puente del Común, Autopista Norte de Bogotá, D.C. \\ Apartado 140013, Colombia \\ Jorge E. Pulido, Álvaro Ramírez, Luz E. Higuita, Sandra M. Arias, Carlos S. Gonzáles y Leonidas J. Ruiz \\ Escuela de Ingeniería Química, Universidad Industrial de Santander, Bucaramanga, Colombia \\ Recebido em 28/5/09; aceito em 9/11/09; publicado na web em 23/3/10
}

\begin{abstract}
POLYURETHANE ELASTOMERS FROM CASTOR OIL AND CHEMICALLY MODIFIED YUCCA STARCH: SYNTHESIS AND PHYSICAL-CHEMICAL, PHYSICAL-MECHANICAL AND THERMICAL PROPERTIES. Chemical modification of cassava starch was conducted through an acylation reaction by using pyridine and propionic anhydride to replace the functional groups of starch. Polyurethane elastomers were prepared using suspensions of the mixture obtained from castor oil and yucca starch that was modified by a propionic anhydride reaction. The suspensions were characterized by means of tests based on The Fourier Transform Infrared Spectroscopy and the Hydroxyl Index. The castor oil-AMP suspensions were used for the PU synthesis. The PUs were characterized by their physical-mechanical properties like tension- deformation and Shore A. hardness, thermal gravimetric analysis and swelling test. The density cross-linking of from swelling tests was determined by applying the Flory-Rehner equation.
\end{abstract}

Keywords: polyurethane elastomers; cassava starch; acylation.

\section{INTRODUCCIÓN}

La síntesis de elastómeros de poliuretano (PU) y el estudio de sus propiedades ha recibido notable atención. Recientes desarrollos de mezclas de polímeros con almidón modificado y sin modificar muestran que las propiedades de los PUs cambian debido a la incorporación de esta molécula en la matriz de PU. ${ }^{1}$ Desai y colaboradores utilizaron el almidón como agente entrecruzante en elastómeros de PU. ${ }^{2}$ El principal problema que hallaron al adicionar almidón al PU fue el carácter hidrofílico de la molécula en contraste con el carácter hidrofóbico de la estructura de PU. Este contraste produce una baja adhesión interfacial entre los gránulos de almidón y la matriz de PU. Santayanon y Wootthikanokkhan incorporaron almidón de yuca modificado por reacción de acilación con anhídrido propiónico a un sistema de PU previamente sintetizado. ${ }^{3}$ Este estudio reveló que la molécula de almidón modificado tuvo mayor afinidad con la estructura de PU de carácter hidrofóbico, debido a que al modificar el almidón los grupos hidroxilo de carácter hidrofílico presentes cambiaron a grupos éster de carácter hidrofóbico.

Trabajos previos realizados en el grupo de investigación utilizando almidón de yuca sin modificar en sistemas de poliuretano obtenidos a partir del aceite de ricino original y modificado por transesterificación, revelaron que el esfuerzo máximo y la elongación de ruptura aumentan. Este efecto se debe primordialmente a que el almidón actuó como relleno reforzante del material. Es decir, la densidad de entrecruzamiento no varía, por que el almidón refuerza la red pero no hace parte de ella. ${ }^{4-6}$

En este trabajo se modificó el almidón de yuca por medio de una reacción de acilación, usando como reactivo anhídrido propiónico y piridina como catalizador. Las variables del diseño fueron la temperatura de preactivación, el tiempo de preactivación y la temperatura de acilación. El proceso se llevó a cabo usando un diseño de experimentos factorial $2^{3}$. El objetivo de la modificación fue sustituir los hidrógenos presentes en los grupos hidroxilo de la molécula de la

\footnotetext{
*e-mail: manuel.valero@unisabana.edu.co
}

amilosa por uno de los grupos propionil del anhídrido propiónico en la reacción de acilación. El almidón original $(\mathrm{S})$ y el almidón modificado (AMP) se mezclaron con el aceite de ricino (CO) hasta obtener una suspensión. Estas suspensiones se emplearon en la síntesis de los PU. Al modificar los grupos hidroxilos del almidón por grupos ésteres las propiedades de los PU variaron. Se buscó comprender cuál es el efecto de la modificación de la estructura química del almidón por acilación sobre las propiedades fisicomecánicas, térmicas y fisicoquímicas de los elastómeros de PU y compararlos con los homólogos obtenidos a partir de suspensiones aceite-almidón original, en función de la adhesión interfacial entre el gránulo de almidón original y modificado y la matriz de poliuretano.

\section{EXPERIMENTAL}

\section{Materiales}

Aceite de ricino (índice de hidroxilo: $163 \mathrm{mg} \mathrm{KOH} / g$ e índice de acidez: 2,33 $\mathrm{mg} \mathrm{KOH} / \mathrm{g}$ ) grado USP, distribuido por laboratorios León, Bucaramanga, Colombia. Pentaeritritol marca Fisher, distribuido por Produquímica de Colombia S.A. El almidón utilizado fue almidón de yuca, marca Maicena distribuido por Disa en Cali, Colombia. Los reactivos utilizados para la caracterización fueron de grado analítico marca Aldrich, distribuidos por Arquilab Ltda.

\section{Métodos}

\section{Modificación de la estructura química del almidón por acilación}

Para llevar a cabo la modificación del almidón de yuca, siguiendo el diseño de experimento factorial $2^{3}$, se alternaron tres parámetros en dos valores diferentes cada uno, con una combinación de experimentación que da como resultado ocho pruebas diferentes (Figura 1). ${ }^{7}$ Para la preactivación de la reacción se alternaron la temperatura de preactivación $\left(T_{p}\right)$ y tiempo de preactivación $\left(t_{p}\right)$ y para la reacción de acilación se alternó la temperatura de acilación $\left(T_{A}\right)$. Se mantuvo constante la cantidad de almidón, el volumen de piridina y el volu- 
men de anhídrido propiónico. Para la temperatura de preactivación se utilizaron los valores $T_{P 1}=85^{\circ} \mathrm{C}$ y $T_{P 2}=95^{\circ} \mathrm{C}$, para el tiempo de preactivación se establecieron los valores $t_{P 1}=2 \mathrm{~h}$ y $t_{P 2}=4 \mathrm{~h}$, y la temperatura de acilación tomó los valores $T_{A 1}=65^{\circ} \mathrm{C}$ y $T_{A 1}=75^{\circ} \mathrm{C}$.

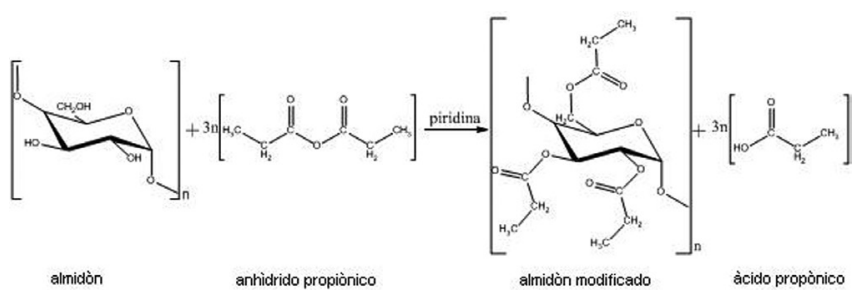

Figura 1. Esquema de la reacción de acilación del almidón de yuca con anhídrido propiónico

En un balón-reactor se agregaron $1.25 \mathrm{~g}$ de almidón de yuca y $10 \mathrm{~mL}$ de piridina con agitación constante y un ambiente inerte generado por la introducción de nitrógeno al balón en el montaje. Se calentó la mezcla a una temperatura de preactivación $T_{P 1}$ durante un tiempo de preactivación $t_{P 1}$. Al finalizar el tiempo de preactivación se agregaron al balón $4.1 \mathrm{~mL}$ de anhídrido propiónico y se cambió la temperatura de preactivación $T_{P 1}$ a la temperatura de acilación $T_{A 1}$. Se continuó agitando y se retiró el nitrógeno del montaje. La reacción se mantuvo a estas condiciones durante $22 \mathrm{~h}$. Después de las $22 \mathrm{~h}$ el balón se desmontó y se le agregaron $50 \mathrm{~mL}$ de isopropanol para disolver la muestra, luego se filtró y se lavó con metanol. La muestra contenida en el papel de filtro se pesó y se secó en una estufa a 343 K durante 24 h. Luego del secado la muestra se pesó. Se utilizó el mismo procedimiento de síntesis para cada uno de los siete experimentos restantes (Tabla 1).

Tabla 1. Porcentaje de propionil y porcentaje de humedad en función de los parámetros de reacción

\begin{tabular}{lccccccc}
\hline Prueba & $\begin{array}{c}\text { Piridina } \\
(\mathrm{mL})\end{array}$ & $\begin{array}{c}\text { Anhídrido } \\
\text { propiónico } \\
(\mathrm{mL})\end{array}$ & $T_{P}\left[{ }^{[} \mathrm{C}\right]^{1}$ & $t_{P}[h]^{2}$ & $T_{A}\left[{ }^{\circ} \mathrm{C}\right]^{3}$ & $\begin{array}{c}\% \\
\text { humedad }\end{array}$ & $\begin{array}{c}\% \\
\text { propionil }\end{array}$ \\
\hline$R_{1}$ & 10 & 4,1 & 85 & 2 & 65 & 41 & 34,2 \\
$R_{2}$ & 10 & 4,1 & 85 & 4 & 65 & 48 & 29,5 \\
$R_{3}$ & $\mathbf{1 0}$ & $\mathbf{4 , 1}$ & $\mathbf{9 5}$ & $\mathbf{2}$ & $\mathbf{6 5}$ & $\mathbf{3 8}$ & $\mathbf{3 7 , 6}$ \\
$R_{4}$ & 10 & 4,1 & 95 & 4 & 65 & 41 & 31,5 \\
$R_{5}$ & 10 & 4,1 & 85 & 2 & 75 & 52 & 26,2 \\
$R_{6}$ & 10 & 4,1 & 85 & 4 & 75 & 56 & 22,1 \\
$R_{7}$ & 10 & 4,1 & 95 & 2 & 75 & 46 & 29,6 \\
$R_{8}$ & 10 & 4,1 & 95 & 4 & 75 & 49 & 27,5 \\
\hline
\end{tabular}

${ }^{1}\left(T_{P}\right)$ : temperatura de preactivación; ${ }^{2}\left(t_{P}\right)$ : tiempo de preactivación; ${ }^{3}\left(T_{A}\right)$ : temperatura de acilación.

El porcentaje de humedad de la muestra se calculó de la siguiente manera:

$\%$ humedad $=\frac{W_{H}-W_{S}}{W_{H}} \times 100$

donde $W_{H}$ : peso de la muestra antes del secado y $W_{S}$ : peso de la muestra después del secado.

El porcentaje de humedad es una manera de comprobar que tanto almidón fue modificado. A menor porcentaje de humedad menor capacidad del propianato del almidón de absorber agua.

Con el fin de obtener el porcentaje de propionil de la muestra de almidón modificado: se tomó una muestra de $0.5 \mathrm{~g}$ y se disolvió en $10 \mathrm{~mL}$ de metanol. Se preparó una solución $1 \mathrm{~mol} / \mathrm{L}$ de $\mathrm{NaOH}$ y una solución $1 \mathrm{~mol} / \mathrm{L}$ de $\mathrm{HCl}$. A la muestra disuelta se le agregaron 10 $\mathrm{mL}$ de solución de $\mathrm{NaOH}$ para saponificar el éster. La base en exceso se determinó por titulación con la solución de $\mathrm{HCl}$ y fenolftaleína como indicador. El porcentaje de propionil indica que tantos grupos hidroxilos fueron sustituidos.

El porcentaje de propionil se obtuvo de la siguiente manera:

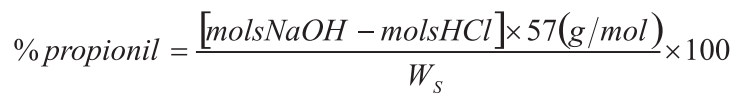

Preparación de suspensiones aceite de ricino-almidón de yuca modificado con anhídrido propiónico en presencia de un catalizador

Se realizó en un balón-reactor de 3 bocas equipado con sensor de temperatura, agitador magnético de $200 \mathrm{rpm}$, atmósfera inerte de nitrógeno y condensador de reflujo. El aceite de higuerilla se cargó y se mantuvo a $100{ }^{\circ} \mathrm{C}$ durante 10 min para retirar las trazas de humedad. La temperatura se elevó lentamente a $130^{\circ} \mathrm{C}$ y posteriormente se adicionó los gránulos de AMP y óxido de plomo como catalizador $(\mathrm{PbO})$ con relación $0.05 \%$ (masa $\mathrm{PbO} /$ masa de aceite). El proceso de incorporación de AMP se llevó a cabo a $210 \pm 5{ }^{\circ} \mathrm{C}$ por $2 \mathrm{~h}$. El contenido de AMP en las suspensiones aceite-AMP fue de 0, 3, 5 y $10 \%$ con relación masa de AMP/volumen de suspensión.

Se determinó el índice de hidroxilo de las suspensiones obtenidas según la norma ASTM D1957-86, con el fin de medir de medir el aumento en el número de grupos $\mathrm{OH}$ de los diferentes polioles obtenidos a partir de la modificación del almidón de yuca. El índice de hidroxilo se calculó según la Ecuación 3:

Valor hidroxilo $=\frac{B+\left(\left(S^{*} A\right) / C\right)-V}{S} N * 56.1 \quad[m g \mathrm{KOH} / \mathrm{g}$ muestra $](3)$

$A=$ Solución de $\mathrm{KOH}$ requerida para la titulación del valor ácido $(\mathrm{mL}) ; B=$ Solución de $\mathrm{KOH}$ requerida para la titulación del blanco $(\mathrm{mL}) ; C=$ Muestra usada para el valor ácido $(\mathrm{g}) ; V=$ Solución de $\mathrm{KOH}$ requerida para la titulación de la muestra acetilada $(\mathrm{mL}) ; S=$ Muestra usada para la acetilación (g); $N=$ Normalidad de la solución.

Sintesis de poliuretanos a partir de las suspensiones aceitealmidón y aceite-AMP

La suspensión aceite/almidón se mezcló con el diisocianato de difenilmetano (MDI), que fue previamente fundido a $50{ }^{\circ} \mathrm{C}$, con una la relación $\mathrm{NCO} / \mathrm{OH}=1$. La mezcla se agitó vigorosamente. $\mathrm{Al}$ prepolímero obtenido se le aplicó a vacío para retirar las burbujas de aire y luego se vertió en un molde de placas paralelas. El periodo de curado constó de dos etapas, la primera a temperatura ambiente durante 4 h y la segunda a $90{ }^{\circ} \mathrm{C}$, también durante $4 \mathrm{~h}$.

\section{Caracterización de elastómeros de poliuretano}

El comportamiento térmico de las muestras se investigó por termogravimetría (la masa de una sustancia es medida como una función de la temperatura mientras la sustancia es sujeta a un programa de temperatura controlada). El análisis termogravimétrico se llevó a cabo en una termobalanza TA 2050 TGA (Universidad Industrial de Santander, Colombia). La velocidad de calentamiento fue de $10^{\circ} \mathrm{C} /$ min en un intervalo de temperatura entre $25-700{ }^{\circ} \mathrm{C}$. Los ensayos se realizaron bajo atmósfera de nitrógeno. ${ }^{8,9}$

Las pruebas de resistencia a tensión se realizaron en una máquina de tensión universal Instron (Universidad Industrial de Santander, Colombia). Se utilizaron mordazas cilíndricas que sostenían la muestra. La velocidad de prueba fue $2.5 \mathrm{~cm} / \mathrm{min}$ a una temperatura $19 \pm$ $2{ }^{\circ} \mathrm{C}$, según la norma ASTM D638. Las dimensiones de la muestra fueron láminas de $40 \mathrm{~mm} * 6 \mathrm{~mm} * 3 \mathrm{~mm}$ (largo*alto*ancho). Para 
cada muestra de 3 a 5 replicas fueron evaluadas en las mismas condiciones. La dureza de los materiales se determinó según la norma ASTM D785. Se determinó utilizando un durómetro shore A con punzón, y se tomaron medidas en al menos 10 puntos dentro de la lámina del material. ${ }^{10,11}$

La densidad de entrecruzamiento de algunos poliuretanos sintetizados se determinó por ensayos de hinchamiento. Muestras cilíndricas de dimensiones $1.6 \pm 0.2 \mathrm{~cm}$ de diámetro y $0.3 \pm 0.1 \mathrm{~cm}$ de espesor fueron puestas acetona (solvente en el cual los materiales presentaron el máximo hinchamiento en las pruebas de resistencia al ataque químico durante 7 días a $27 \pm 2{ }^{\circ} \mathrm{C}$ ). Después de lograr el equilibrio (punto en el cual el grado de hinchamiento no cambia) se removió el exceso de solvente y se determinó el cambio de volumen en las muestras. La densidad de entrecruzamiento de los poliuretanos se obtuvo usando la siguiente Ecuación de Flory-Rehner. ${ }^{12-14}$

$$
\frac{v c}{V o}\left(\mathrm{~mol} / \mathrm{cm}^{3}\right)=\frac{-2 *\left[\mathrm{v}+\chi_{12} * \mathrm{v}^{2}+\ln (1-\mathrm{v})\right]}{V_{1} *\left[2 * \mathrm{v}^{1 / 3}-\mathrm{v}\right]}
$$

$v c=$ Número efectivo de cadenas entrecruzadas; $V_{1}=$ Volumen molar del solvente; $x_{12}=$ Parámetro de interacción solvente-polímero; $V=V_{0} / V$ donde $V_{0}=$ Volumen del polímero sin hinchar (seco) y $V=$ Volumen del polímero hinchado en el punto de equilibrio.

\section{RESULTADOS}

\section{Porcentaje de propionil y porcentaje de humedad}

En la Tabla 1 se muestra el porcentaje de propionil y el porcentaje de humedad para cada experimento. Se observa que el menor porcentaje de humedad y mayor porcentaje de propionil lo registró el experimento $R_{3}$. Una disminución del porcentaje de humedad refleja un mayor grado de sustitución de los grupos hidroxilo por grupos propionil. Según los resultados obtenidos las condiciones de reacción más favorables para la modificación del almidón con anhídrido propiónico son las registradas en el experimento $R_{3}$, reportadas en la Tabla 1.

\section{Espectroscopia infrarroja de transformada de Fourier (FTIR)}

En la Figura 2 se muestra el espectro del almidón puro y el espectro del AMP. Se observa en el espectro del AMP un pico de fuerte intensidad a una longitud de onda de $1750 \mathrm{~cm}^{-1}$ que corresponde a la banda de absorción del grupo éster, mientras que el espectro del almidón original no la registra. Se observa también que la banda correspondiente a los grupos $\mathrm{OH}$ a una longitud de onda de 3100-3700 $\mathrm{cm}^{-1}$ disminuye la intensidad en el espectro del AMP en comparación al almidón puro. Este efecto se debe a que al modificar el almidón puro por reacción de acilación se reemplazan los grupos hidroxilos de la molécula de almidón por grupos ésteres.

En la Figura 3 se observan los espectros FTIR del aceite de ricino y las suspensiones aceite-AMP. No se presentaron cambios en ninguna de las bandas de absorción. Las bandas corresponden a los grupos $\mathrm{OH}\left(3429-3377 \mathrm{~cm}^{-1}\right), \mathrm{CH}\left(3006-2854 \mathrm{~cm}^{1}\right)$, éster $\left(1743 \mathrm{~cm}^{-1}\right)$ y $\mathrm{CH}_{2}-\mathrm{CH}_{3}\left(1454 \mathrm{~cm}^{-1}\right)$. Al incorporar el AMP al aceite de ricino a través de una mezcla física no ocurre cambios en la estructura. Este resultado valida los datos obtenidos del índice de hidroxilo de las suspensiones aceite-AMP.

\section{Índice de hidroxilo}

En la Figura 4 se muestra la relación existente entre el índice de hidroxilo de las suspensiones y el agente modificador (almidón puro

\section{ALMIDÓN PURO}


Figura 2. Espectros FTIR y estructuras químicas del a) almidón puro y b) AMP

y AMP). No se encontró una diferencia estadísticamente significativa en el índice de hidroxilo de las suspensiones aceite-AMP. En contraste se observó un aumento en el índice de hidroxilo de suspensiones aceite-almidón. Este resultado se debe a que al modificar el almidón por acilación se reemplazan los grupos hidroxilos por grupos ésteres. Por lo tanto, disminuye la funcionalidad hidroxílica en comparación con el almidón original.

ACEITE DE HIGUERILLA

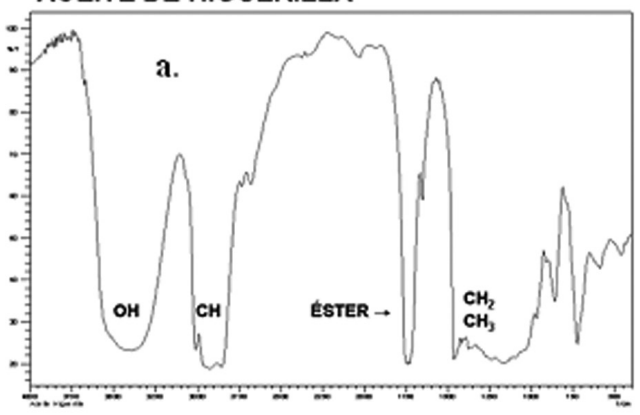

SUSPENSIÓN (S5)

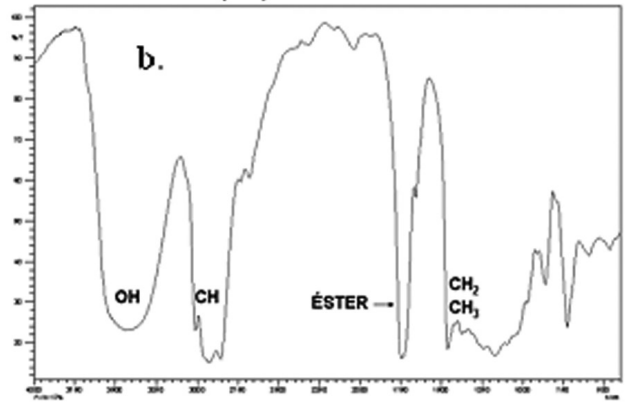

Figura 3. Espectros FTIR: a) aceite de ricino y b) suspensión aceite-AMP 


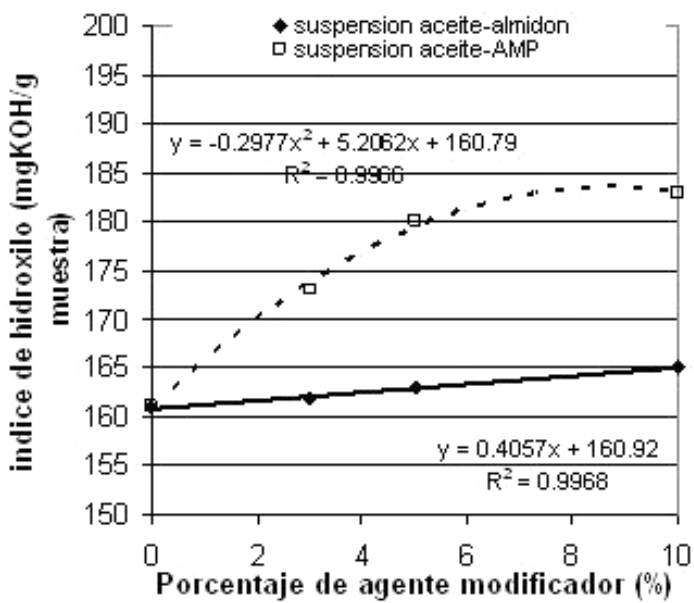

Figura 4. Índice de hidroxilo de las suspensiones aceite-AMP y suspensiones aceite-almidón

\section{Caracterización de los elastómeros de poliuretano}

Los PU sintetizados a partir de suspensiones aceite-AMP presentaron tres zonas de degradación. Las temperaturas de descomposición se registran en la Tabla 2. La primera zona comprendida entre 210-330 ${ }^{\circ} \mathrm{C}$ presentó una pérdida de $20 \%$ en peso del material. Correspondiente a la pérdida de hidrocarburos alifáticos lineales del aceite de higuerilla. La segunda zona comprendida entre $330-450^{\circ} \mathrm{C}$ corresponde a la volatilización de los grupos presentes en las moléculas que forman los enlaces úretanos (isocianatos y ésteres que no reaccionaron). En la tercera zona entre $450-579{ }^{\circ} \mathrm{C}$ se dio la descomposición de las redes de uretano. Estas tres zonas de degradación son similares a las presentadas por los PU homólogos a partir de suspensiones aceitealmidón. Los resultados anteriores validan la conjetura de que al adicionar almidón original y modificado, el efecto principal es actuar como agente reforzante de la red de poliuretano.

Tabla 2. Temperaturas de descomposición de los PU obtenidos a partir de suspensiones aceite-AMPa

\begin{tabular}{lcccc}
\hline $\begin{array}{l}\text { Porcentaje de } \\
\text { pérdida de peso } \\
(\%)\end{array}$ & \multicolumn{4}{c}{ Temperaturas de descomposición } \\
\hline 10 & PU-P0 AMP & PU-P3 AMP & PU-P5 AMP & PU-P10 AMP \\
60 & $430 \pm 2$ & $210 \pm 2$ & $209 \pm 2$ & $315 \pm 2$ \\
90 & $550 \pm 2$ & $560 \pm 2$ & $579 \pm 2$ & $546 \pm 2$ \\
$\begin{array}{l}\text { Porcentaje de } \\
\text { residuo a 500 }\end{array}$ & 1.385 & 0.087 & 1.497 & 0.594 \\
${ }^{\circ} \mathrm{C}(\%)$ & & & & \\
\hline
\end{tabular}

${ }^{\mathrm{a}} \mathrm{El}$ análisis termogravimétrico se llevó a cabo en una termobalanza TA 2050 TGA. La velocidad de calentamiento fue de $10^{\circ} \mathrm{C} / \mathrm{min}$ en un intervalo de temperatura entre $25-700^{\circ} \mathrm{C}$. Los ensayos se realizaron bajo atmósfera de nitrógeno.

En la Tabla 3 se observa los valores del módulo de Young, la tensión de ruptura, la elongación y la dureza shore A en función del porcentaje de agente modificador incorporado para los PU obtenidos de suspensiones aceite-AMP y suspensiones aceite-almidón. También se observa que el incremento en el esfuerzo último y dureza shore A es mayor para los PU a partir de aceite-AMP que para los homólogos obtenidos a partir de aceite-almidón puro, mientras que la elongación de ruptura disminuye para los materiales obtenidos a partir de suspensiones aceite-AMP.

En la Figura 5 se observa un aumento en la densidad de entrecruzamiento en función del porcentaje de agente modificador adicionado.
Tabla 3. Propiedades mecánicas de los poliuretanos obtenidos a partir de las suspensiones aceite-almidón original y aceite-AMP

\begin{tabular}{lcccccc}
\hline & \multicolumn{2}{c}{ PU (Aceite-Almidón original) } & \multicolumn{3}{c}{ PU (Aceite-AMP) } \\
\hline$\%^{\mathrm{a}}$ & $\sigma(\mathrm{KPa})^{\mathrm{b}}$ & $\in(\%)^{\mathrm{c}}$ & $\begin{array}{r}\text { Dureza } \\
\text { Shore A }\end{array}$ & $\sigma_{(\mathrm{KPa})^{\mathrm{b}}} \in(\%)^{\mathrm{c}}$ & $\begin{array}{c}\text { Dureza } \\
\text { Shore A }\end{array}$ \\
0 & 1800 & 128 & 48 & 1800 & 128 & 48 \\
3 & 1934 & 137 & 61 & 2203 & 121 & 69 \\
5 & 2153 & 141 & 64 & 3232 & 100 & 75 \\
10 & 2509 & 155 & 70 & 3300 & 58 & 84 \\
\hline
\end{tabular}





Figura 5. Densidad de entrecruzamiento de los PU obtenidos a partir de suspensiones aceite-AMP y suspensiones aceite-almidón

El aumento en la densidad de entrecruzamiento es mayor para los PU obtenidos a partir de suspensiones aceite-AMP que para los materiales obtenidos a partir de suspensiones aceite-almidón.

\section{DISCUSIÓN}

Los resultados mostraron que los $\mathrm{PU}$ obtenidos a partir de suspensiones aceite-AMP poseen un mayor esfuerzo último y dureza shore A, mientras que la elongación de ruptura disminuye, en comparación con las propiedades de los PU obtenidos a partir de las suspensiones aceite-almidón sin modificar. Estos resultados se deben al cambio en las interacciones que existen entre la matriz de PU y el almidón sin modificar y modificado. Cuando se utilizó el almidón original, los enlaces tipo puentes de hidrógeno ocurren entre los grupos $\mathrm{OH}$ del almidón y los grupos carbonilos del grupo uretano. Mientras que, cuando se utilizó AMP los puentes de hidrógeno ocurren entre los grupos carbonilos del AMP y los grupos $\mathrm{N}-\mathrm{H}$ del grupo uretano (Figura 6). ${ }^{15,16}$ Las fuerzas intermoleculares presentes entre los grupos se calcularon por la ley de Coulomb basada en los enlaces-longitud y la relación de las fuerzas de van der Waals entre dos moléculas polares. ${ }^{11}$ Para el cálculo se tuvo en cuenta el momento dipolar $(\mu$ $=$ Momento dipolar (D): indicador de la polaridad general de una molécula) del grupo éster $(\mu=2 \mathrm{D})$, grupo $\mathrm{OH}(\mu=1.5 \mathrm{D})$ y se estimó el momento dipolar del grupo uretano $(\mu=3.5 \mathrm{D})$. La fuerza de atracción que existe entre la matrizdePUyelAMP $\left(F=3.706 * 10^{-42} \frac{C^{2}}{\mathrm{~A}^{2}}\right)$ es mayor con respecto a la fuerza entre la matriz de PU y el almidón original $\left(F=2.832 * 10^{-42} \frac{C^{2}}{\mathrm{~A}^{2}}\right)($ Carga $=(\mathrm{C})$ y Radio de enlace $(\AA)) \cdot \mathrm{Al}$ 
aumentar la polaridad entre los grupos funcionales crece la afinidad entre la matriz de PU y el AMP, por lo que las cadenas se atraen y el volumen libre del polímero disminuye en comparación con el polímero sintetizado a partir del almidón original. Los resultados obtenidos de la variación de la densidad de entrecruzamiento determinada a partir de ensayos de hinchamiento validan este comportamiento.

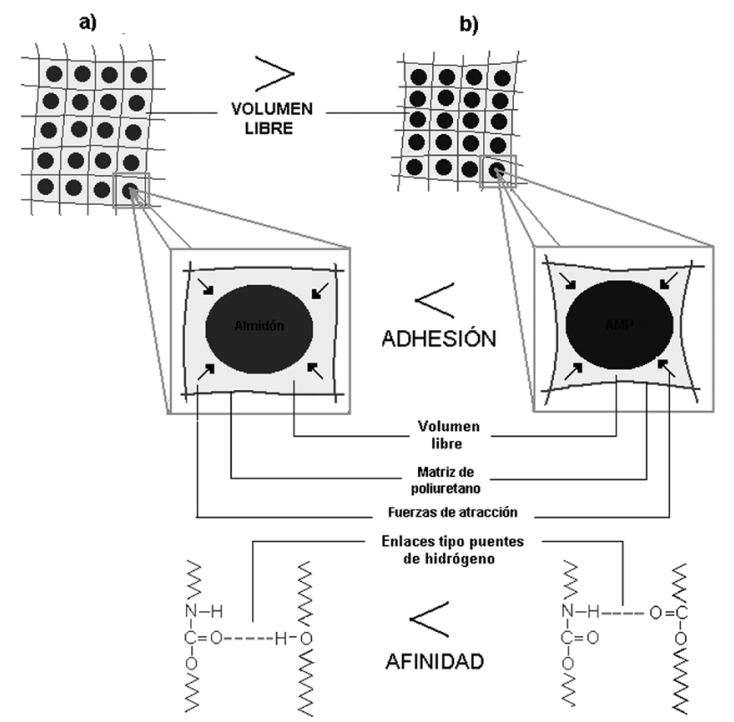

Figura 6. Esquema de la adhesión interfacial de la matriz de PU con a) la molécula de almidón, b) la molécula de AMP

En los sistemas de PU obtenidos a partir de suspensiones aceiteAMP se encontró que la disminución en la densidad de entrecruzamiento obedece a un incremento en la adhesión interfacial entre el PU y el AMP. Los resultados encontrados de la densidad de entrecruzamiento a partir de ensayos de hinchamiento de los materiales obtenidos de suspensiones aceite-AMP sustentan el incremento en la adhesión interfacial debido a que la densidad de entrecruzamiento aumentó en función de la concentración de AMP como respuesta a una mayor afinidad y no relacionado con el cambio en el entrecruzamiento de la red polimérica.

La explicación de los resultados de la densidad de entrecruzamiento también se relacionó con el tipo de ensayo que se utilizó para determinarla. Esta prueba es función del volumen libre. Por lo tanto, se encontró que no fue la adecuada para PU sintetizados a partir de almidón modificado y sin modificar que actúan como agente reforzante de la red.

\section{CONCLUSIONES}

Las condiciones más favorables para la modificación el almidón de yuca por acilación con anhídrido propiónico son: la temperatura de preactivación de $95^{\circ} \mathrm{C}$, el tiempo de preactivación de 2 h y la temperatura de acilación de $65^{\circ} \mathrm{C}$. En estas condiciones se alcanzó un porcentaje del $38 \%$ de sustitución de los grupos hidroxilo por los grupos propionil.

Se encontró que no fue posible lograr una incorporación química del AMP al aceite de higuerilla al hacer la suspensión, debido a que no hubo una interacción química entre los grupos hidroxilo del aceite de ricino y los grupos propionato del AMP. Por lo tanto, este actuó como agente reforzante de la red.

Los elastómeros de PU obtenidos de las suspensiones aceite-AMP poseen un mayor esfuerzo último, una mayor dureza y una menor elongación de ruptura que los materiales homólogos obtenidos a partir de suspensiones aceite-almidón. Estas propiedades obedecieron a una mayor adhesión interfacial presente entre los gránulos de AMP (fase dispersa) y la matriz de PU (fase continua), debido a que las fuerzas de atracción (relacionada con la polaridad de las moléculas) son mayores entre el PU y el AMP que para PU y almidón original, bajo las condiciones de síntesis teniendo la misma relación $\mathrm{NCO} /$ $\mathrm{OH}$ e igual porcentaje de agente modificador.

\section{REFERENCIAS}

1. Valero, M.; Pulido, J.; Ramírez, A.; Cheng, Z.; Rev. Polímeros Ciencia y Tecnología 2009, XIX, 21.

2. Desai, S.; Thakore, I.; Sarawade, B.; Devi, S.; Polym. Eng. Sci. 2000, 40, 1200.

3. Santayanon, R.; Wootthikanokkhan, J.; Carbohydr. Polym. 2003, 51, 17.

4. Valero, M.; Pulido, J.; Ramírez, A.; Cheng, Z.; Rev. Iberoamer. Polím. 2007, 8, 203.

5. Somani, K.; Kansara, S.; Parmar, R.; Patel, N.; Int. J. Polym. Mater. 2004, 53, 283.

6. Seung, K. H.; Broecker, H. C.; Macromolecules. Matter. Eng. 2003, 288, 569.

7. Seung, K. H.; Broecker, H. C.; Polymer 2002, 43, 5227.

8. Yongshang, L.; Tighzert, L.; Dole, P.; Erre, D.; Polymer 2005, 46, 9863.

9. Kendaganna, B. K.; Siddaramaiah, S..; J. Appl. Polym. Sci. 2003, 90, 2945.

10. Tharanathan, R. N.; Crit. Rev. Food Sci. Nutrition 2005, 45, 371.

11. Chenal, J-M.; J Widmaier, J-M.; Polymer 2005, 46, 671.

12. Frisch, K. C.; J. Polym. Sci., Part B: Polym. Phys. 2003, 8, 921.

13. Ramis, X.; Cadenato, A.; Morancho, J. M.; Salla, J. M.; Polymer 2001, 42, 9469.

14. Valero, M.; Pulido, J.; Ramírez, A.; Cheng, Z.; J. Am. Oil Chem. Soc. 2009, 86, 383.

15. Vega, J.; Sibaja, M.; Hernández, M.; Alvarado, P.; Rev. Iberoamer. Polím. 2006, 7, 99.

16. Yongshang, L.; Zhang, L.; Zhang, X.; Zhou, Y.; Polymer 2003, 44, 6689. 